\title{
Reply to 'Comment on: The NQO1 polymorphism C609T (Pro187Ser) and cancer susceptibility: a comprehensive meta-analysis'
}

\author{
B Lajin ${ }^{*}, 1$ and A Alachkar ${ }^{2}$
}

\section{${ }^{1}$ Department of Analytical Chemistry, Faculty of Pharmacy, University of Aleppo, Aleppo, Syria and ${ }^{2}$ Department of Pharmacology, School of} Medicine, University of California Irvine, Irvine, CA, USA

Sir,

We have strictly followed the published general guidelines for conducting meta-analyses (Stroup et al, 2000; Minelli et al, 2009). Therefore we tested for deviations from HWE and reported in our paper the deviation of five studies from HWE. Deviation from HWE may imply genotyping error and/or possible heterogeneity in the control population. Most of the excluded studies strongly violated HWE with $P$-values $=0$. Nevertheless, when the five studies were included in the meta-analysis the association remained statistically significant with almost unaltered odds ratios. For example, for the TT vs CC model $\mathrm{OR}=1.17(1.06-1.30), P=0.002$, compared to the reported results with the exclusion of the five studies, $\mathrm{OR}=1.18(1.07-1.31), P=0.002$. This is not unexpected given the large number of studies included and the fact that the majority of studies were in compliance with the HWE principle. This confirms that no bias has been introduced by the exclusion of the five studies and that the concluded positive association between total cancer risk and the investigated polymorphism is unaffected.

The distinction between the source of controls was not made unambiguously in all published papers. Therefore, we avoided stratification according to the source of controls. In addition, we believe that such stratification is irrelevant within the context of our meta-analysis and that there is no difference in the genotypic distribution between hospital-based and population-based controls since it is assumed that the control subjects in all studies were not diagnosed with any type of cancer or any other condition commonly associated with the studied polymorphism.

Finally, we believe that it is unlikely that the inadvertently missed singlecase sample had an impact on the results and conclusions of the meta-analysis that included 21178 case samples.

\section{REFERENCES}

Minelli C, Thompson JR, Abrams KR, Thakkinstian A, Attia J (2009) The quality of meta-analyses of genetic association studies: a review with recommendations. Am J Epidemiol 170(11): 1333-1343.

Stroup DF, Berlin JA, Morton SC, Olkin I, Williamson GD, Rennie D, Moher D, Becker BJ, Sipe TA, Thacker SB (2000) Meta-analysis Of Observational Studies in Epidemiology (MOOSE) Group. Meta-analysis of observational studies in epidemiology: a proposal for reporting. JAMA 283(15): 2008-2012.

*Correspondence: Dr B Lajin; E-mail: BassamL7@yahoo.co.uk;

Published online 28 October 2014

(c) 2014 Cancer Research UK. All rights reserved 0007-0920/14

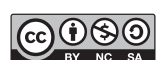

http://creativecommons.org/licenses/by-nc-sa/3.0/

\section{Distinguishing sedentary from inactive: implications for meta-analyses}

B M Lynch ${ }^{*}, 1,2$ and T Boyle ${ }^{3}$

${ }^{1}$ Physical Activity Laboratory, Baker IDI Heart and Diabetes Institute, Melbourne, Victoria, Australia; ${ }^{2}$ Melbourne School of Population and Global Health, The University of Melbourne, Parkville, Victoria, Australia and ${ }^{3}$ Epidemiology Group, Harry Perkins Institute for Medical Research, The University of Western Australia, Perth, Western Australia, Australia

We read with great interest the meta-analysis by Cong et al (2014) recently published in British Journal of Cancer. As the authors acknowledge, sedentary behaviour is distinct from the lack of moderate- to vigorous-intensity physical activity. As the first quantitative review of the studies examining associations of sedentary behaviour on colon and rectal cancer risk, this article makes a timely and novel contribution to the literature. However, we are concerned that the combined risk estimates generated by this meta-analysis may not accurately reflect the effect that can be attributed to sedentary behaviour.

Many of the risk estimates included in the meta-analysis are from studies that investigated the association between occupational physical activity and the risk of colon and/or rectal cancers. As noted by Yates et al (2011), the ordinal scales commonly used to assess occupational physical activity (e.g., 'sedentary', 'moderate, 'high') are not necessarily ordinal scales of sedentary behaviour. As high levels of sedentary behaviour can co-exist with high levels of physical activity, even within specific occupations, using these estimates of occupational physical activity to infer sedentary behaviour is likely to introduce substantial misclassification bias.

A related issue is the inclusion of studies that have classified sedentary behaviour based on job title. While we do not believe it is necessarily wrong to include estimates of sedentary behaviour that are job title based, it is important to note that this method does not take into account within-job variation, seasonal changes or changes in job requirements over time (LaPorte et al, 1985), and may not reflect the actual activities performed on the job (Ainsworth et al, 1999). We would recommend that in future meta-analyses and reviews, these studies be given a lower exposure assessment quality rating than studies using self-reported or objectively assessed measures of sedentary behaviour. In addition, we suggest that subgroup analyses are conducted to investigate whether the results of studies relying on job title-based measures of sedentary behaviour differ from the results of studies with self-reported or objectively assessed measures of sedentary behaviour.

Another issue that arises when using ordinal scales of occupational physical activity (job title-based or self-reported) in a sedentary behaviour context is the selection of the appropriate referent category. The most suitable referent group to compare jobs with high amounts of sedentary behaviour with are jobs that involve 'mostly standing' or 'light' activity. Within the meta-analysis performed by Cong et al (2014), there are several instances where the authors selected the most physically active category as the referent group (Garabrant et al, 1984; Fraser and Pearce, 1993; Weiderpass et al, 2003; Moradi et al, 2008). The relative risks generated by comparing the sedentary category with the most physically active will not solely reflect the effect of sedentary behaviour on colorectal cancer risk; part of the risk estimate will be attributed to the (inverse) of the risk reduction associated with physical activity. A similar error was made with the inclusion of data from two studies that compared recreational sedentary behaviour with recreational physical activity (Thune and Lund, 1996; Colbert et al, 2001).

There are two final points that we would like to raise. First, the risk estimates included in the meta-analysis from the Campbell et al (2013) study pertain to colorectal cancer-specific survival rather than colorectal cancer risk. Second, there are three studies for which the authors have included risk estimates for two different measures of sedentary behaviour (e.g., recreational and occupational sedentary behaviour) in the primary meta-analysis (Thune and Lund, 1996; Colbert et al, 2001; Howard et al, 2008). This is effectively including the same 
study population twice, so these studies are arguably contributing more weight to the overall effect size than appropriate.

Despite the concerns raised, we acknowledge that the meta-analysis presented by Cong et al (2014) has drawn attention to the potential role of sedentary behaviour in colon and rectal cancer aetiology. Clearly further studies, using well-designed and tested measures of sedentary behaviour, are required in this field.

\section{REFERENCES}

Ainsworth BE, Richardson MT, Jacobs Jr DR, Leon AS, Sternfield B (1999) Accuracy of recall of occupational physical activity by questionnaire. J Clin Epidemiol 52: 219-227.

Campbell PT, Patel AV, Newton CC, Jacobs EJ, Gapstur SM (2013) Associations of recreational physical activity and leisure time spent sitting with colorectal cancer survival. J Clin Oncol 31: 876-885.

Colbert LH, Hartman TJ, Malila N, Limburg PI, Pietinen P, Virtamo J, Taylor PR, Albanes D (2001) Physical activity in relation to cancer of the colon and rectum in a cohort of male smokers. Cancer Epidemiol Biomarkers Prev 10: 265-268.

Cong YJ, Gan Y, Sun HL, Deng J, Cao SY, Xu X, Lu ZX (2014) Association of sedentary behaviour with colon and rectal cancer: a meta-analysis of observational studies. Br J Cancer 110: 817-826.
Fraser G, Pearce N (1993) Occupational physical activity and risk of cancer of the colon and rectum in New Zealand males. Cancer Causes Control 4: 45-50.

Garabrant DH, Peters JM, Mack TM, Bernstein L (1984) Job activity and colon cancer risk. Am J Epidemiol 119: 1005-1014.

Howard RA, Freedman DM, Park Y, Hollenbeck A, Schatzkin A, Leitzmann MF (2008) Physical activity, sedentary behaviour, and the risk of colon and rectal cancer in the NIH-AARP Diet and Health Study. Cancer Causes Control 19: 939-953.

LaPorte RE, Montoye HJ, Caspersen CJ (1985) Assessment of physical activity in epidemiologic research: problems and prospects. Public Health Rep 100: $131-146$

Moradi T, Gridley G, Bjork J, Dosemeci M, Ji BT, Berkel HJ, Lemeshow S (2008) Occupational physical activity and risk for cancer of the colon and rectum in Sweden among men and women by anatomic subsite. Eur J Cancer Prev 17: 201-208.

Thune I, Lund E (1996) Physical activity and risk of colorectal cancer in men and women. Br I Cancer 73: 1134-1140.

Weiderpass E, Vainio H, Kauppinen T, Vasama-Neuvonen K, Partanen T, Pukkala E (2003) Occupational exposures and gastrointestinal cancers among Finnish women. J Occup Environ Med 45: 305-315.

Yates T, Wilmot EG, Davies MJ, Gorely T, Edwardson C, Biddle S, Khunti K (2011) Sedentary behaviour: what's in a definition? Am J Prev Med 40: e33-e34.

*Correspondence: Dr BM Lynch; E-mail: brigid.lynch@bakeridi.edu.au Published online 25 February 2014

(c) 2014 Cancer Research UK. All rights reserved 0007 - 0920/14

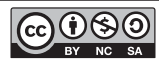

http://creativecommons.org/licenses/by-nc-sa/3.0/

\title{
Reply: Comment on 'Association of sedentary behaviour with colon and rectal cancer: a meta- analysis of observational studies'
}

\author{
Z Lu*, ${ }^{*}, \mathrm{~S} \mathrm{Cao}^{1}, \mathrm{Y} \mathrm{Gan}{ }^{1}$ and Y Cong
}

\section{${ }^{1}$ School of Public Health, Tongji Medical College, Huazhong University of Science and Technology, Wuhan, Hubei, China}

We would like to thank Dr. Lynch and Boyle (2014) for their valuable comments and suggestions on our meta-analysis 'Association of sedentary behaviour with colon and rectal cancer: a meta-analysis of observational studies' (Cong et al, 2014). This meta-analysis made a timely and novel contribution to the literature about associations of sedentary behaviour on colon and rectal cancer risk. Although some imperfection may exist, they did not materially influence our result. Now, we are replying to the main comments mentioned by Lynch and Boyle.

Indeed, sedentary behaviour is distinctly different from occupational sedentariness and the lack of moderate- to vigorous-intensity physical activity. But in the included original studies, these exposures are difficult to be strictly differentiated. In our initial manuscript, we only focused on self-reported order to avoid missing more relevant studies, we took into account the sedentary behaviour that is measured by job title-based response in the revised manuscript. Now, we did a subgroup analysis by types of assessment of sedentary behaviour, and the result showed that there was no substantial difference in the two types of measure of sedentary behaviour. For colon cancer, the pooled OR of sedentary behaviour measured by job title-based response was 1.39 ( $\left.95 \% \mathrm{CI}, 1.20-1.60, I^{2}=63.7 \%\right)$, whereas the pooled OR of self-reported sedentary behaviour was 1.27 (95\% CI, $\left.1.18-1.36, I^{2}=26.7 \%\right)$. sedentary behaviours. On the basis of the suggestion of one of the reviewers, in

The difference between them was insignificant $(P$ for interaction $=0.289)$. For rectal cancer, the pooled OR of sedentary behaviour measured by job titlebased response was $1.11\left(95 \% \mathrm{CI}, 1.03-1.20, I^{2}=4.2 \%\right)$, whereas the pooled OR of self-reported sedentary behaviour was 1.01 (95\% CI, $0.92-1.11$, $I^{2}=19.7 \%$ ). The difference between them was insignificant too ( $P$ for interaction $=0.156$ )

There are three studies that we included twice because the authors reported the risk estimates for two different measures of sedentary behaviour. Indeed, this may be contributing more (although not much more) weight to the overall effect size, but including only one of the two measures of sedentary behaviour is also inappropriate.

In summary, we appreciate most of Lynch and Boyle's comments and suggestions. Our meta-analysis indeed has some flaws, but these defects do not alter our main results and conclusions.

\section{REFERENCES}

Cong YJ, Gan Y, Sun HL, Deng J, Cao SY, Xu X, Lu ZX (2014) Association of sedentary behaviour with colon and rectal cancer: a meta-analysis of observational studies. $\mathrm{Br} J$ Cancer 110: 817-826.

Lynch BM, Boyle T (2014) Distinguishing sedentary from inactive: implications for meta-analyses. Br J Cancer 111: 2202-2203.

${ }^{\star}$ Correspondence: Professor Z Lu; E-mail: zuxunlu @yahoo.com Published online 25 February 2014

(c) 2014 Cancer Research UK. All rights reserved 0007-0920/14

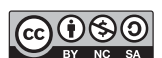

http://creativecommons.org/licenses/by-nc-sa/3.0/
$\mathrm{BJC}$ OPEN 1

\section{Coexistence of KRAS mutation with mutant but not wild-type EGFR predicts response to tyrosine-kinase inhibitors in human lung cancer}

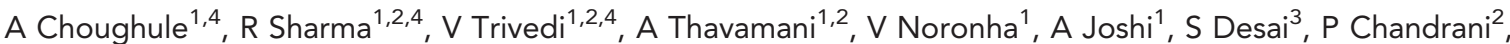
P Sundaram ${ }^{2}$, S Utture ${ }^{1}$, N Jambhekar ${ }^{3}$, S Gupta ${ }^{1}$, J Aich ${ }^{*}, 2$, K Prabhash $^{*, 1}$ and A Dutt ${ }^{\star}, 2$

${ }^{1}$ Department of Medical Oncology, Tata Memorial Hospital, Tata Memorial Center, Mumbai, India; ${ }^{2}$ Advanced Centre for Treatment, Research and Education in Cancer, Tata Memorial Center, Navi Mumbai, India and ${ }^{3}$ Department of Pathology, Tata Memorial Hospital, Tata Memorial Center, Mumbai, India

Sir,

EGFR and KRAS mutations occur mutually exclusively in NSCLC, suggesting functional redundancy (Kosaka et al, 2004; Pao et al, 2005;
Shigematsu et al, 2005; Tam et al, 2006). However, they predict contrasting response rates to tyrosine-kinase inhibitors (TKIs) - while EGFR mutation predicts longer progression-free survival rate (Lynch et al, 2004; 